\title{
Características académicas, cognoscitivas y emocionales de estudiantes universitarios de primer ingreso
}

\author{
Daniel González Lomelí* \\ Sandra Castañeda Figueiras** \\ María de los Ángeles Maytorena Noriega*
}

\section{Resumen}

La presente investigación se llevó a cabo con el propósito de evaluar los indicadores académicos y psicológicos que poseen los estudiantes de nuevo ingreso a la Universidad de Sonora, durante su primer ciclo escolar universitario. Se seleccionó de forma aleatoria a estudiantes de 11 licenciaturas en cinco unidades regionales de la institución, con el fin de identificar su perfil de ingreso en función de las respuestas dadas a una serie de instrumentos de medición, en los que se evalúan variables tales como el uso de estrategias de autorregulación y de certeza vocacional.

La muestra comprende un total de 425 estudiantes distribuidos en las diferentes unidades académicas de esta universidad, así mismo, se seleccionaron en la Unidad Regional Norte, las carreras de Mercadotecnia y Químico Biólogo en el campus de Caborca, y dentro de esa mis unidad, pero en el campus Nogales a la licenciatura en Comunicación Organizacional; en la Unidad Regional Centro se seleccionaron las carreras de Economía, Trabajo Social, Lingüística, Tecnología Electrónica, Medicina e Ingeniería Civil y en la Unidad Regional Sur (campus Navojoa) fueron las carreras de Administración e Ingeniería en Sistemas de Información las seleccionadas.

Los resultados reflejan la necesidad de preparar a los estudiantes de forma directa o a través de los maestros en el desarrollo de estrategias de autorregularización que los lleven a un aprendizaje efectivo, esto, en combinación con una orientación a sus metas personales y académicas, como estudiantes universitarios y futuros profesionales de la disciplina en la que se están formando.

\footnotetext{
Abstract

In order to evaluate some of the academic and psychological indicators have new students to college in northwest Mexico, during their first academic school year is randomly selected students from 11 degrees in five regional units the institution to identify the income profile depending on the answers to a series of measurement instruments that assess variables such as the use of self-regulation strategies and career certainty. A total of 425 students majoring in Marketing and Biological Chemistry campus Caborca, Hermosillo on campus were selected races of Economics, Social Work, Linguistics, Electronics Technology, Medical and Civil Engineering; Navojoa on campus belong to the races administration and Information Systems Engineering and the Nogales campus bachelor's degree in Organizational Communication. The results reflect the need to prepare students directly or through teachers in the development of self-regulation strategies that will lead to effective learning in combination with an orientation to their personal and academic goals, as college students and future professional discipline in which they are forming.
}

\footnotetext{
*Universidad de Sonora

**Universidad Nacional Autónoma de México
}

Palabras clave: autorregulación, certeza vocacional, evaluación de estudiantes, contexto universitario. 
En este reporte se presentan los resultados de la investigación sobre las variables certeza vocacional y uso de estrategias de autorregulación y cómo afectan al desempeño académico en el marco de la medición cognitiva del aprendizaje y la identificación de los factores que inciden en el aprendizaje complejo.

Dentro del concepto de Medición Cognitiva del Aprendizaje, Castañeda (2002) enfatiza la importancia de las características y ventajas de los sistemas orientados cognitivamente frente a los sistemas educativos no orientados cognitivamente y señala que los productos del aprendizaje son concebidos, dentro de esta perspectiva, como "desarrollos graduales de habilidad cognitiva" (p. 64), de ahí que ubicar dónde se encuentra el estudiante en su proceso de aprendizaje, a partir de un modelo de desarrollo de habilidad cognoscitiva, sea necesario para la prescripción de experiencias de aprendizaje a la medida, a partir de proveer información diagnóstica.

Se puede afirmar que la medición de la calidad de los resultados de aprendizaje es una forma de medición cognoscitiva dado que el producto que el examinando genera cuando se enfrenta a los reactivos es lo que el examinador usa como evidencia para determinar el logro alcanzado. La investigación ha establecido que la cognición siempre es un proceso situado a un dominio -lo que cualifica, en la práctica, a un dominio de contenido específico (como puede ser el de la medicina, el de la ingeniería, el derecho o el de la psicología)- es su extrema dependencia de sus particularidades, haciendo necesario atender no nada más al conocimiento involucrado, sino también, a las demandas en las cuales la cognición situada toma lugar.

En el caso de la Universidad de Sonora la estrategia de admisión de estudiantes a los diferentes programas de licenciatura ha tenido varias versiones, desde la aceptación de los estudiantes en forma masiva, hasta la aplicación del Examen de Habilidades y Conocimientos Básicos (EXHCOBA) de
Backhoff y Tirado (1994), pasando por cursos propedéuticos, donde los estudiantes tienen su primer acercamiento a la forma en que se lleva a cabo el currículo localmente, a través de pláticas y conferencias que los profesores sostienen con los aspirantes a ingresar a las diversas licenciaturas.

El proceso de ingreso a la Universidad de Sonora incluye, además del promedio de bachillerato y la evaluación de conocimientos disciplinares, la aplicación del Examen de Habilidades y Conocimientos Básicos (EXHCOBA) de Backhoff y Tirado (1992) instrumento elaborado para "evaluar los conceptos estructurales y destrezas cognitivas que dan soporte al conocimiento que un estudiante debe tener antes de ingresar al nivel de educación superior" (p. 50) su principal objetivo es predecir el éxito académico de los estudiantes universitarios de nuevo ingreso, así como diagnosticar el nivel de escolaridad básica que poseen.

Desde hace tiempo existen estudios que informan sobre la calidad de la ejecución de los estudiantes al responder al EXHCOBA. Por ejemplo, González (2002) afirma que los aspirantes a ingresar a una licenciatura en psicología, que presentaron el examen en el año de 1996, lograron obtener sólo $48 \%$ de aciertos en promedio; así mismo, observó una relación negativa directa entre el grado de dificultad de los contenidos medidos y el porcentaje de aciertos. Es decir, a mayor dificultad de los contenidos evaluados, menos aciertos. Otros autores (Bachkoff \& Tirado, 1993) obtuvieron resultados similares en otra universidad del noroeste de México.

Durante el ciclo escolar 1997-1998 González (op.cit.) refiere que la ejecución de los solicitantes a ingresar a alguna de las licenciaturas de la División de Ciencias Sociales, fue por debajo del $50 \%$ de aciertos en el EXHCOBA, mientras que González-Lizárraga (1999) identifica que para los semestres 1997-1 al 1998-2 las mejores ejecuciones en el EXHCOBA 
fueron para los aspirantes a la licenciatura en Física (más de $70 \%$ de aciertos), seguidos por los estudiantes interesados en cursar la licenciatura en Literaturas Hispánicas (61.67\% de aciertos) y en tercer lugar los estudiantes que aplicaron para ingresar a la licenciatura en Enseñanza del Inglés, con casi $60 \%$ de aciertos. Uno de los trabajos más recientes (Piña-Osuna, 2011) utilizando el EXHCOBA, indica que la calificación promedio, en las áreas de Conocimientos Básicos de Especialidad que mide dicho examen, de los estudiantes que ingresaron en el semestre 2009-2 a seis licenciaturas de una universidad pública del noroeste de México, muestra que la licenciatura con mejor puntaje promedio logró 143.91 (84.62\% de un máximo de 170 de calificación) y que la licenciatura con el puntaje más bajo alcanzó una calificación de 77.93 , sólo un poco más del $45 \%$ de la puntuación posible.

También existen reportes del índice de excelencia en la formación a nivel de licenciatura, medido por las calificaciones obtenidas en las asignaturas. Por ejemplo, González (2002) refiere que en el período de 1984-1993, sólo $13 \%$ de los estudiantes de la licenciatura en Psicología se ubicaba en el nivel de excelencia (90 a 100 de calificación) y que para el período de 1994 a 1999 el porcentaje de calificaciones de excelencia fue similar (11.4\%). Regresando al estudio de Piña-Osuna (op. cit.) y en relación a la calidad de la formación de los estudiantes universitarios, es importante resaltar que los estudiantes de las seis licenciaturas lograron un promedio de calificaciones de 86.95 en el primer semestre y al finalizar el segundo semestre el promedio bajó a 79.39, es decir, se obtuvo un promedio general durante el primer año de 83.17 de calificación; de esta manera, la licenciatura con menor promedio de calificación (75.05) al primer año, fue la misma que obtuvo la menor puntuación en el examen de admisión.

Hasta aquí, los datos presentados muestran una situación poco satisfactoria, del perfil de ingreso y de formación de los estudiantes que conformaron las muestras de los estudios referidos, a partir de los conocimientos y habilidades adquiridos en los subsistemas educativos previos al ingreso a la universidad y en la ejecución académica en las asignaturas correspondientes a las disciplinas en las que se están formando.

También se han realizado investigaciones sobre variables asociadas al desempeño académico con estudiantes de la Universidad de Sonora sobre certeza vocacional (González \& Maytorena, en prensa; González \& Maytorena, 2005), estrategias de aprendizaje (González, Castañeda \& Corral, 2002; González, Castañeda \& Maytorena, 2000) y perspectiva temporal y morosidad (González, Maytorena, Lohr \& Carreño, 2006). Además, la literatura nacional e internacional ha permitido identificar, que el desempeño académico de los estudiantes universitarios, recibe efectos de la variable promedio de bachillerato, así como de las variables certeza vocacional (Aguilar, Peña, Pacheco \& De la Paz, 1993), estrategias cognoscitivas de aprendizaje (Castañeda \& Ortega, 2004), autorregulación (Castañeda \& Martínez, 1999; Pintrich, 1998; Pintrich \& García, 1991), perspectiva temporal (Corral et al. 2003; Díaz-Morales, 2006; Zimbardo \& Boyd, 1999) y de la morosidad académica (Aguilar \& Valencia, 1994; Aguilar, Valencia, Martínez \& Vallejo, 2002), entre otras variables.

Respecto a la variable certeza vocacional, esta ha sido definida por Aguilar (1992) como la seguridad que el estudiante tiene en la carrera que ha elegido, y está conformada por cinco factores: autoestima (es la evaluación característica que hace el sujeto de sí mismo como persona y juega un importante papel en la implementación de autoconcepto vocacional); ansiedad en la elección de carrera (es el grado de ansiedad alcanzada en el proceso de toma de decisiones); indecisión generalizada (que es la incapacidad que tienen las personas para tomar decisiones); información de carrera (es la necesidad de recolectar datos y experiencia 
real de las ocupaciones antes de tomar una decisión vocacional); y autoconocimiento (es la necesidad que tiene cada persona de definirse a sí misma y descubrirse, es importante en los modelos de elección de carrera para conocer sus cualidades, habilidades e intereses).

Una manera que se ha desarrollado para evaluar la certeza vocacional en estudiantes tanto universitarios como de bachillerato, es a través del uso de inventarios tales como el Inventario Ampliado de Factores de Carrera (IAFC), el cual posee dos versiones, uno para bachillerato y otro para educación superior.

EI IAFC es un cuestionario de auto-reporte adaptado a población mexicana por Aguilar et al. (1992) el cual mide comportamientos y percepciones del estudiante sobre las dimensiones cognoscitivas de autoconocimiento, información sobre la carrera y autoeficacia, las cuales se registran en un formato tipo Likert y las escalas emocionales de ansiedad en la elección de carrera e indecisión generalizada son medidas mediante diferencial semántico. También posee una escala de certeza vocacional utilizada como medida de contraste.

González y Maytorena (2005) aplicaron el IAFC a 229 estudiantes de primer semestre de la Universidad de Sonora, los factores de carrera explicaron el $10 \%$ de la varianza del esfuerzo académico y el $33 \%$ de la varianza de la seguridad vocacional. Posteriormente, Maytorena, González y Velarde (2006) en un estudio con 490 estudiantes de educación superior, encontraron que las puntuaciones medias de las escalas del IAFC y la alta certeza vocacional que logran los estudiantes de la muestra, contrasta con la alta necesidad de autoeficacia y la alta necesidad de información de carrera que reportan.

Otro estudio interesado en las variables referidas al desempeño académico es el de Araujo, Cerezo, Coronado y Hernández (2008) quienes realizaron un estudio con universitarios en relación con la ansiedad escolar y la autoevaluación con el propósito de indagar la relación entre el desempeño académico de estudiantes universitarios con la tendencia a presentar conductas emocionales de ansiedad en el ámbito escolar, valoradas a través de un procedimiento conductual computarizado con monitoreo de reactividad fisiológica. De manera adicional se exploró la relación del desempeño académico con el auto-concepto, entendido como el auto-reporte sobre intención de superación académica, un índice subjetivo de aprendizaje y la auto-evaluación sobre el desempeño académico final.

La investigación educativa se ha abocado también a descifrar los métodos involucrados en el proceso de aprendizaje, lo que ha conducido al hecho de reconocer la importancia de no centrarse únicamente en los componentes cognoscitivos implicados en el aprendizaje, sino también atender los componentes afectivos o motivacionales (Rinaudo, Chiecher y Donolo, 2003) involucrados en el proceso de aprender.

Suárez y Fernández (2005) plantean que estudiar las estrategias motivacionales dentro del proceso de aprendizaje es un tema de actualidad que hemos superado a nivel teórico, aunque no ha repercutido lo necesario en su investigación, sobre todo si se toma en cuenta que el tipo de aprendizaje que los estudiantes deben desarrollar está en manos de su capacidad de esfuerzo, lo cual conduce a la necesidad de estar lo suficientemente motivado y desarrollar conductas metacognoscitivas.

Lo anterior lleva a hablar del proceso de autorregulación, el cual se concibe como un proceso interactivo complejo que involucra no sólo autorregulación metacognitiva, sino también metamotivacional y ambas pueden ser enseñadas vía el modelamiento que ofrezca el docente (Boekaerts, 1995 en Castañeda y Ortega 2004). Los dos tipos de autorregulación se entrelazan y afectan la inversión del esfuerzo del estudiante, por una parte; y la calidad de ejecución en la tarea de aprendizaje, por la otra. Según 
Castañeda y Ortega (2004) tanto el conocimiento autorregulatorio como la operación de las habilidades de autorregulación, permiten que el estudiante pueda establecer la modificación, selección o construcción de las estrategias necesarias para el logro de las metas deseadas.

Se han realizado investigaciones internacionales (Carbonero y Navarro, 2006; Pintrich, 1998; De Corte, 1999), nacionales (Castañeda, 1998; Aguilar, Valencia, Martínez y Vallejo, 2002) y regionales (González, 2002) con el fin de conocer cómo es que los estudiantes aprenden, procesan y adquieren la información aprendida y la mayoría de ellas esbozan la necesidad de atender y evaluar los procesos motivacionales y de autorregulación en el aprendizaje, sea en tareas generales o en tareas específicas.

En México se desarrolló un instrumento que mide el uso de estrategias cognoscitivas, metacognoscitivas y metamotivacionales de aprendizaje, el cual se deriva del modelo de enseñanza y aprendizaje estratégico elaborado por Castañeda (1998). Tal modelo propone la evaluación y fomento de las estrategias cognoscitivas de aprendizaje así como también de las estrategias metacognoscitivas y metamotivacionales; en cuanto a la autorregulación Castañeda clasifica el estudio, fomento y evaluación de la autorregulación considerando tres componentes fundamentales: el aprendiz, en cuanto a su eficacia percibida; su autonomía percibida; su contingencia interna; y su aprobación externa; así mismo, la tarea en cuanto a la tarea en sí y en cuanto a su orientación al logro y los materiales de aprendizaje (Castañeda, Lugo, Pineda y Romero, 1998). El instrumento de evaluación derivado de este modelo es el Inventario de estrategias de estudio y autorregulaciónIEEA (Castañeda, 2003) utilizado en esta investigación.

Los estudios se realizaron atendiendo a las implicaciones éticas y metodológicas. En cuanto a los aspectos éticos se observará el Código
Ético del Psicólogo (Sociedad Mexicana de Psicología, 2010) en lo referente a los apartados de la Calidad de la Valoración y/o Evaluación Psicológica (Arts. 15, 16, 17, 18, 20, 21, 27), el Consentimiento Informado (Arts. 118, 122 y 123), la Confidencialidad (Arts. 132 y 133), la Comunicación de Resultados (Arts. 50, 51, 52, $53,55,56,57,58,59$ y 60 ) y la Confidencialidad de los Resultados (Arts. 61, 67, 68 y 69).

\section{Estudio 1}

El propósito de este estudio fue evaluar el perfil de ingreso y la certeza vocacional en estudiantes universitarios, el cual presenta el análisis de las características académicas que los estudiantes poseen al solicitar su ingreso a la universidad, así como la identificación de su perfil vocacional durante el primer semestre en la licenciatura $y$, por último, se analizan los indicadores de desempeño académico al terminar su primer año en la licenciatura respectiva.

\section{Método}

\section{Participantes}

Se seleccionó al azar una licenciatura por cada una de las 11 divisiones que forman a las unidades regionales (centro, norte y sur) de la Universidad de Sonora: a) en las licenciaturas cuya población de primer ingreso fue menor a 100, se incluyó en la muestra al total de los estudiantes $y, b)$ en las licenciaturas cuya población de primer ingreso fue mayor a 100, se seleccionaron al azar 100 estudiantes.

La muestra quedó integrada por 423 estudiantes, de los cuales $58.39 \%$ estudiaban en la Unidad Regional Centro, $22.93 \%$ en la Unidad Regional Norte (Caborca y Nogales) y $18.67 \%$ en la Unidad Regional Sur (Navojoa). Del total de estudiantes $53 \%$ son mujeres, $78 \%$ solteros y $81 \%$ no trabajan. La edad promedio de los estudiantes de la muestra fue de 19.8 años (d. e. $=3.17$ años). El ingreso familiar mensual reportado 
fue de $\$ 10,282.77$ (d. e. $=18,286.821$ ). Respecto a cursos y materia de orientación vocacional $44 \%$ de la muestra refiere haber llevado una asignatura de orientación vocacional cuando cursaban el bachillerato, mientras que sólo $11 \%$ señala haber llevado un curso de orientación vocacional. La distribución de la muestra por licenciatura se presenta en la tabla 1.

Tabla 1. Distribución de la muestra por licenciatura

\begin{tabular}{lcc}
\hline Licenciatura & Frecuencia & $\%$ \\
\hline Unidad regional centro & 19 & 4.49 \\
Tecnología electrónica & 59 & 13.94 \\
Ing. civil & 60 & 14.18 \\
Medicina & 48 & 11.34 \\
Trabajo social & 40 & 9.45 \\
Economía & 21 & 4.96 \\
Lingüística & & \\
& & 5.67 \\
Unidad regional norte & 24 & 8.27 \\
Mercadotecnia & 35 & 8.98 \\
Químico biólogo clínico & 38 & \\
Comunicación organizacional & & 15.36 \\
\hline Unidad regional sur & & \\
Administración de empresas & 65 &
\end{tabular}

\section{Instrumentos}

Los estudiantes respondieron el Inventario Ampliado de Factores de Carrera, IAFC (Aguilar et al., 1992), en su versión para población universitaria. Los autores (op. cit.) reportaron coeficientes de confiabilidad (alfa de Cronbach mayores a .77) y validez concurrente del inventario con estudiantes de tres facultades de una universidad pública de la ciudad de México. El IAFC está constituido por tres escalas de información, tipo Likert: información sobre la carrera, autoconocimiento y autoeficacia; así mismo, por dos escalas emocionales, tipo diferencial semántico: indecisión generalizada y ansiedad en la elección de carrera. Además, incluye una escala tipo Likert para medir seguridad vocacional.

1) Ejemplo de reactivos de la dimensión de autoconocimiento:

Para que pueda decidir si sigo o no estudiando la carrera que actualmente estudio, todavía necesito contestar la siguiente pregunta: ¿Cuáles son mis metas específicas en la vida?

2) Ejemplo de reactivos de la dimensión de información sobre la carrera:

Para que pueda decidir si sigo o no estudiando la carrera que actualmente estudio, todavía necesito averiguar qué oportunidades de trabajo ofrece actualmente y a futuro.

3) Ejemplo de reactivos de la dimensión de autoeficacia:

Creo tener la capacidad necesaria para seguir con la carrera que estoy estudiando.

4) Ejemplo de reactivos de la dimensión de seguridad vocacional:

La carrera que actualmente estudio la continuaré hasta terminarla.

5) Ejemplo de reactivo de la dimensión ansiedad en la elección de carrera.

Cuando pienso en si sigo o no estudiando la carrera que actualmente estudio me siento:

a) tenso _ _ _ _ _ relajado

6) Ejemplo de reactivos de la dimensión Indecisión generalizada.

En general tomar decisiones me resulta:

a) confuso claro

Para obtener los puntajes en habilidades y conocimientos básicos se utilizó el Examen de Habilidades y Conocimientos Básicos, EXHCOBA (Bachkoff y Tirado, 1994) que es el examen de ingreso aplicado en la Universidad de Sonora a todos sus aspirantes.

Los integrantes de la muestra también respondieron una Ficha de datos sociodemográficos, para obtener información acerca de la edad, sexo, aspectos de decisión 
vocacional, actividad laboral, número de miembros de la familia, ingreso familiar, ocupación y estudios tanto del padre como de la madre.

\section{Procedimiento}

Se consultaron los expedientes de los participantes en el archivo escolar para obtener información acerca del promedio, materias aprobadas y reprobadas (correspondientes a los primeros y segundos semestres cursados). EI IAFC y la ficha de datos sociodemográficos se aplicaron en el salón de clase, de manera grupal e informada, previa instrucción (indicando que su participación sería voluntaria y confidencial); se inició con la ficha de datos sociodemográficos, posteriormente el IAFC. La aplicación del EXHCOBA quedó a cargo de la Dirección de Servicios Escolares, ya que es un proceso automatizado.

\section{Análisis de datos}

Se realizaron análisis de frecuencia para las variables descriptivas sociodemográficas de género, estado civil, materias reprobadas, escuela de procedencia, cursos y materias de orientación recibidas, situación del estudiante (regular/irregular) y educación y ocupación de los padres; así como, medias con sus respectivas desviaciones estándar para edad y promedio general. Se obtuvieron medias y desviaciones estándar para las variables continuas: número de horas de trabajo, edad y promedios. El estadístico alpha de Cronbach se empleó para analizar la consistencia interna de los instrumentos y medidas de los instrumentos utilizados. También se realizaron comparaciones de medias para muestras independientes para determinar el perfil de los estudiantes de la muestra en cuanto a las variables medidas tales como puntuación en el EXHCOBA, factores de carrera y certeza vocacional. Finalmente, se realizó una prueba no paramétrica alternativa al análisis de varianza que se utiliza para comparar varias muestras independientes (Kruskall-Wallis) tanto de los niveles de certeza vocacional como de las variables de ingreso por licenciatura.

\section{Resultados}

Respecto al bachillerato de procedencia, el mayor porcentaje de estudiantes egresó del Colegio de Bachilleres (28\%); le siguió el sistema federal CBTIS (16.6\%); el tercer lugar lo ocupan otros subsistemas con $15.2 \%$; posteriormente las escuelas particulares (11.8\%); y con menos estudiantes $(9.1 \%)$ se ubica el subsistema CECYTES.

\section{Indicadores académicos de ingreso}

En cuanto a los indicadores de desempeño académico que se consultaron para esta investigación, los valores más altos tanto en promedio de bachillerato, como en aciertos en el EXHCOBA, el puntaje general en el EXHCOBA y el promedio de semestre, fueron para los estudiantes de Medicina (MED). El segundo lugar para el promedio de bachillerato, aciertos en el EXHCOBA y puntaje general en el EXHCOBA fue para los estudiantes de Ingeniería Civil (IC). Dentro de las carreras con más bajos valores se encuentran la Licenciatura en Administración (LA) con un promedio en bachillerato de 78.68 de calificación, un promedio de 72.85 aciertos en el EXHCOBA y 44.62 de promedio en la puntuación general en el EXHCOBA; el promedio menor de primer semestre fue para los estudiantes de la Licenciatura en Tecnología Electrónica (LTE).

En la figura 1 se muestra que en relación a las variables de ingreso a la universidad tales como el promedio de bachillerato y la puntuación en el EXHCOBA, tanto general como de aciertos y errores de los estudiantes, el promedio de bachillerato es la variable más constante entre carreras; los estudiantes de menor puntuación en esta variable son los de 


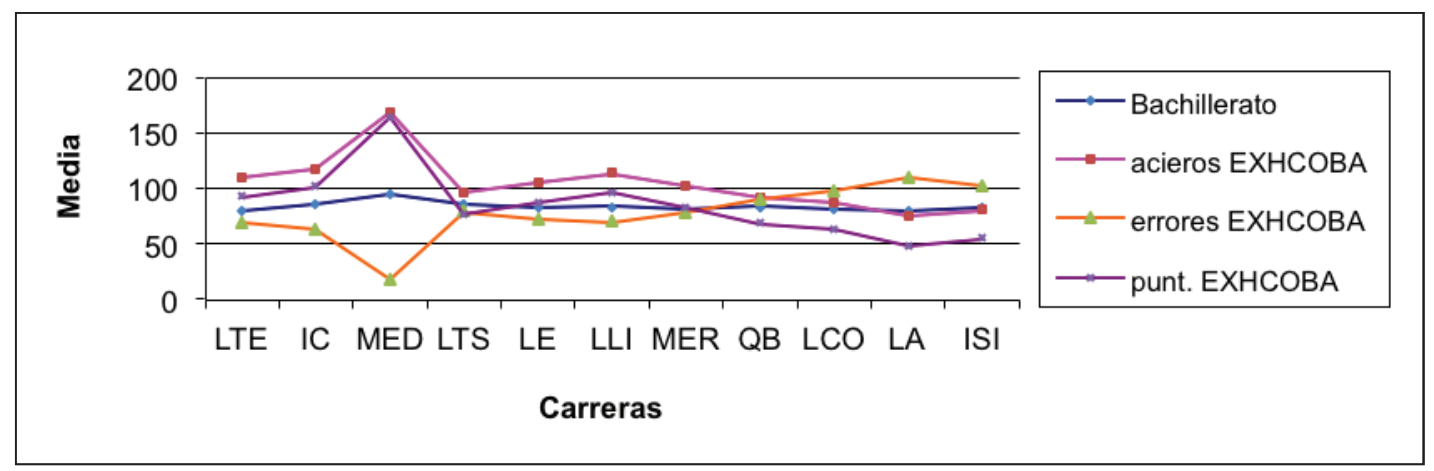

Figura 1. Perfil de variables de ingreso.

Tecnología Electrónica (LTE) y la puntuación más alta fue para los estudiantes de Medicina (MED), donde casi se llega a una media de 100. Respecto a los aciertos, se obtuvieron puntuaciones mayores en los estudiantes de Medicina (MED) quienes superan una media de 165 aciertos; la media menor fue para Administración (LA) con una media inferior a los 100 aciertos. En el caso de los errores en el EXHCOBA ocurre una situación inversamente proporcional, y para la puntuación general en el EXHCOBA se encontró un rango de entre 50 y 100 puntos con excepción de Medicina (MED) quienes superan los 150 puntos.

\section{Perfil de certeza vocacional de los estudiantes}

En cuanto al perfil de certeza vocacional, en la figura 2 se presentan las proporciones de los factores de carrera de cada licenciatura que componen la muestra. En cuanto a las escalas cognoscitivas, los estudiantes de Tecnología Electrónica (LTE), los de Lingüística (LLI) y los de Administración de Empresas (LA) fueron los estudiantes con las puntuaciones más bajas; y quienes tuvieron puntuaciones más altas en estas mismas escalas fueron los estudiantes de Medicina (MED), Mercadotecnia (MER) e Ingeniería Civil (IC). En cuanto a las escalas emocionales, quienes reportan mayor nivel de indecisión generalizada fueron los estudiantes de Ingeniería en Sistemas de la Información (ISI) y Comunicación Organizacional (LCO); mientras que para el nivel de ansiedad ante la elección de carrera el punto más alto se ubica también en Ingeniería en Sistemas de Información (ISI) seguida de los estudiantes de Trabajo Social (LTS).

Una comparación de medias entre los las unidades se realizó a fin de contrastar las diferencias que existen en la certeza vocacional;

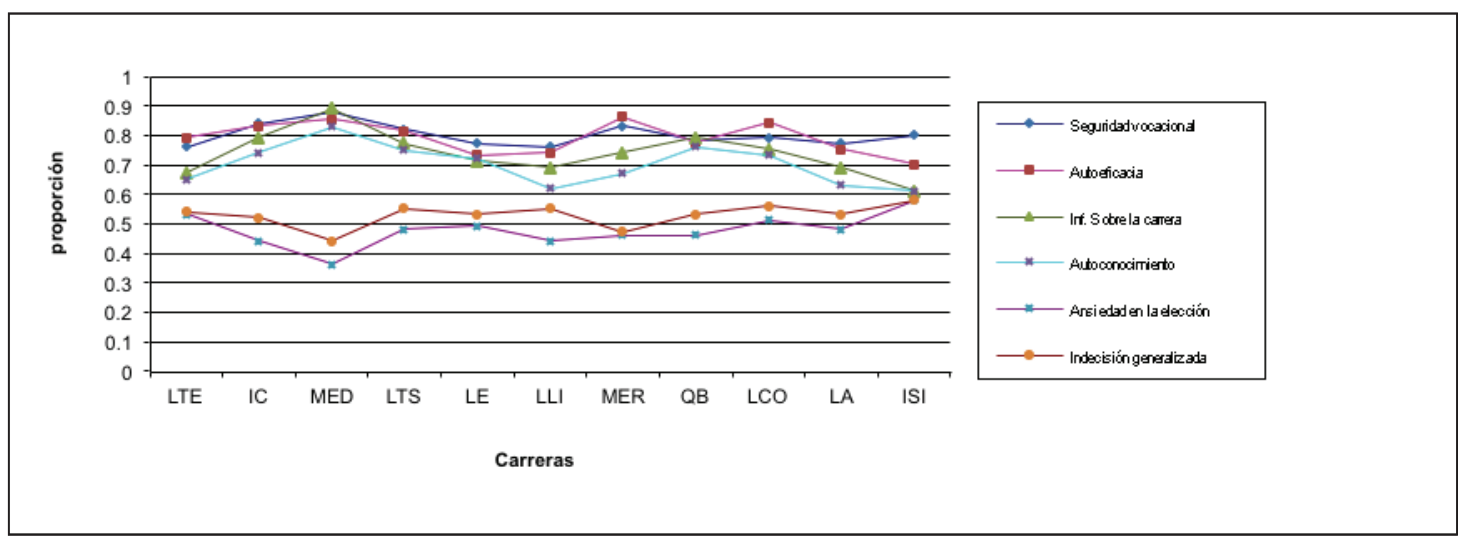

Figura 2. Perfil de certeza vocacional por carrera. 


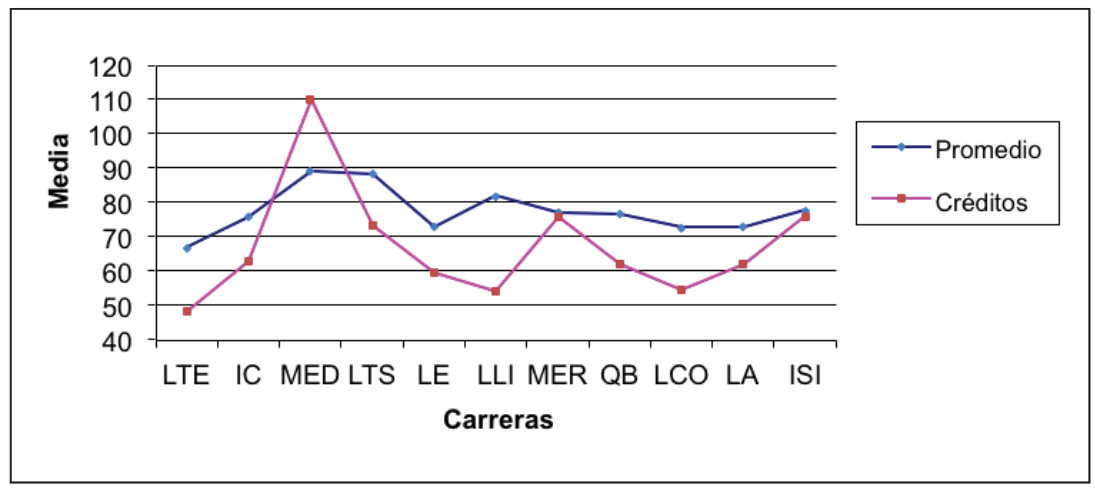

Figura 3. Perfil de indicadores de desempeño académico.

los resultados muestran diferencias significativas a favor de la Unidad Centro, también en las escalas información sobre la carrera y autoconocimiento; y a favor de las unidades foráneas, en la escala ansiedad ante la elección de carrera.

\section{Desempeño académico en el primer ciclo escolar}

Acerca del promedio y los créditos obtenidos por los estudiantes, la figura 3 muestra que los promedios mayores al primer año se ubican entre los estudiantes de Medicina (MED) y Trabajo Social (LTS); mientras que los menores se encuentran en Tecnología Electrónica (LTE) y Comunicación Organizacional (LCO). Respecto al número de créditos acumulados durante el primer año de estudios, los estudiantes que cubrieron mayor número de créditos fueron los de Medicina (MED), Mercadotecnia (MER) e Ingeniería en Sistemas de Información (ISI); mientras que los estudiantes con menores puntuaciones en créditos resultaron ser los de Tecnología Electrónica (LTE), Lingüística (LLI) y Comunicación Organizacional (LCO).

Finalmente, se realizó una prueba no paramétrica alternativa al análisis de varianza que se utiliza para comparar varias muestras independientes (Kruskall-Wallis) para las variables de ingreso, el resultado se presenta en la figura 4. Las carreras con menor puntuación en el EXHCOBA fueron las que corresponden a la Unidad Sur; Administración (LA), Ingeniería en Sistemas de Información (ISI), Medicina (MED) e Ingeniería Civil (IC) de la Unidad Centro, obtuvieron la puntuación más alta en dicha variable. En cuanto al promedio de bachillerato, la carrera con estudiantes de menor promedio fue LTE seguida de LA de la Unidad Sur; las carreras que presentan promedios más altos son, de nuevo, MED e IC; también con distancias superiores a los

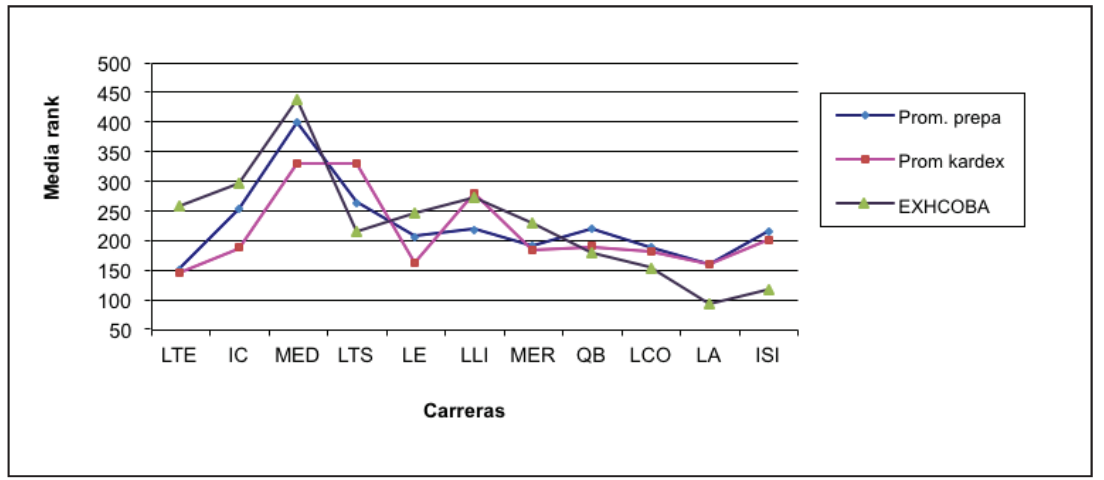

Figura 4. Puntuación media rank de variables de ingreso y desempeño académico. 
100 puntos entre una y otra carrera. El mayor promedio (Kárdex) hasta el primer año cursado fue para MED, seguido de LTS y los promedios menores se ubican en LTE y Economía (LE) de la Unidad Centro y LA de la Unidad Sur.

\section{Discusión}

Los estudiantes que participaron en este estudio poseen niveles altos en certeza vocacional y en autoeficacia; al respecto, Canto y Rodríguez (2000) encontraron en estudiantes de bachillerato, que las puntuaciones de autoeficacia se correlacionan de manera positiva con las puntuaciones de elección de carrera. Esta información fue confirmada por González y Maytorena (2003) y posteriormente por Maytorena, González y Velarde (2006). Sin embargo, se encontraron puntuaciones bajas en las escalas autoconocimiento e información sobre la carrera, escalas que también se han identificado como variables importantes en la elección de carrera.

Cupani y Pérez (2006) elaboraron un estudio para conocer la relación de la elección de carrera con los intereses vocacionales, la autoeficacia y los rasgos de personalidad; la autoeficacia explicó $19 \%$ de la varianza de las intenciones de la elección de carrera. Respecto al autoconocimiento, estos mismos autores indican que incrementar el autoconocimiento ayuda a relacionar sus potencialidades con las ocupaciones o las carreras a fin de realizar una adecuada elección. Los resultados encontrados en México con el IAFC y particularmente los realizados con estudiantes de la Universidad de Sonora también señalan que la autoeficacia y el autoconocimiento son factores fundamentales en la elección de carrera.

Enestudiantessegurosvocacionalmente tampoco se esperan valores bajos de autoconocimiento como ocurrió con estudiantes de bachillerato, donde el autoconocimiento resultó un factor importante para la certeza vocacional después de la información de carrera
(Velarde, González \& Maytorena, 2006) o de indecisión generalizada a áreas no académicas como se ha encontrado en este estudio. Del mismo modo Aguilar, Valencia y Martínez (1998) en Aguilar et al., (2002) encontraron una influencia predominante de la autoeficacia, la motivación de logro y la certeza vocacional sobre la motivación intrínseca, lo cual es un indicador de que las creencias en las propias capacidades, aunado al uso de estrategias de estudio eficaces, son determinantes importantes del interés y dedicación en los estudios.

Ahora bien, es ampliamente documentado que las deficiencias académicas que se encuentran en los estudiantes de la muestra, no son exclusivas de la Universidad de Sonora ni del país; sin embargo, esta situación no puede pasarse por alto dado que el problema pasa del contexto universitario al laboral, por lo que se insiste en atender las debilidades que los estudiantes poseen al ingresar a la educación superior; en el caso de la certeza vocacional, por ejemplo, es necesario que la función del orientador sea valorada no sólo por los estudiantes, sino también por las instituciones educativas que los contratan, pero no les asignan tareas relacionadas con la elección de carrera y el uso de estrategias de aprendizaje en los estudiantes; o bien, aquellas instituciones que han optado por no contratar orientadores educativos, situación que prevalece desde niveles educativos iniciales.

Aunado a lo anterior, está el hecho de que históricamente existen deficiencias en desempeño académico, reprobación y deserción en los estudios de prácticamente todos los niveles educativos existentes; tales índices poco alentadores no pueden ser ajenos a las condiciones bajo las cuales se otorga el ingreso a la escuela, a las condiciones del aprendizaje, a los materiales de estudio y a las prácticas para evaluar los desempeños (López-Olivas, 2007).

Así, la función de los exámenes de admisión en opinión de López-Olivas (2007) es la asignación de lugares disponibles y a quién 
asignarlos, y no la de evaluar los conocimientos ni las habilidades requeridas para una profesión determinada. Tal situación ha generado que los estudiantes sean pasivos y dependientes de la información que otros, como el profesor, les proporcionan, sin generar las habilidades requeridas para continuar aprendiendo y que el aprendizaje sea efectivo.

Cabe comentar lo citado por Abril, Román y Cubillas (2002) al hablar de un período de riesgo al terminar la educación básica y durante el inicio de la educación media. Indican que la deserción en ese período de estudios puede tener relación con la carencia de metas y proyectos de vida del adolescente y con las relaciones familiares. Además, reflejan la necesidad de trabajar en el establecimiento y cumplimiento de metas con los estudiantes universitarios desde que ingresan a la universidad con el fin de que su estancia durante los estudios de licenciatura esté orientada hacia el cumplimiento y logro de las metas establecidas.

\section{Estudio 2:}

En este estudio se determinó el perfil que poseen los estudiantes de primer ingreso a la universidad en lo que se refiere al uso de estrategias de autorregulación y comparar dichos perfiles entre estudiantes de la capital del estado de Sonora con los estudiantes de otras unidades de la Universidad de Sonora en el Estado.

\section{Método}

\section{Participantes}

Participaron en este estudio los mismos 425 estudiantes que lo hicieron en el estudio anterior y de las licenciaturas ya enunciadas y presentadas en la tabla 1.

\section{Instrumentos y medidas}

Dichos estudiantes respondieron también el Inventario de Estrategias de Estudio y Autorregulación, IEEA de Castañeda (2003), el cual contiene 91 reactivos distribuidos en cuatro escalas y 13 subescalas, con cuatro opciones de respuesta tipo likert (desde muy en desacuerdo hasta muy de acuerdo), acerca del uso de estrategias de adquisición de información, recuperación de lo aprendido, procesamiento de información y autorregulación (autorregulación de persona, autorregulación de tarea y autorregulación de materiales). En este estudio se utilizó sólo la escala de autorregulación, de la cual a continuación se presentan ejemplos de reactivos:

1) Autorregulación dimensión persona. Regula la efectividad de la actividad que proviene del aprendiz mismo.

Reactivo: me siento seguro de mis conocimientos y habilidades en la mayoría de mis materias (mide eficacia percibida).

Reactivo: puedo cambiar mi manera de estudiar, sin el apoyo de otros (contingencia interna).

Reactivo: sin importar la naturaleza de la tarea que debo realizar me empeño en ella hasta resolverla o terminarla (autonomía percibida).

Reactivo: me esfuerzo en el estudio, sólo si me reconocen mis mejores amigos (aprobación externa).

2) Autorregulación: dimensión tarea. Estrategias que se dirigen a la tarea respecto a identificar su dificultad, interés en la misma, motivación por la búsqueda del dominio o la búsqueda de aprobación.

Reactivo: tomo decisiones que favorecen lograr mis metas de aprendizaje (logro de metas).

Reactivo: planeo mis actividades de estudio de acuerdo con el nivel de dificultad de la tarea a realizar (tarea en sí).

3) Autorregulación: dimensión de materiales. Autopercepción de qué tan útil resulta un material, facilidad o dificultad de su comprensión y mantenimiento del interés en el mismo.

Reactivo: sé cuándo, dónde y con quién obtener el material que requiero para estudiar.

\section{Análisis de datos}

Se realizaron análisis de comparación de medias para la determinación del perfil y una prueba no paramétrica alternativa al análisis de varianza que se utiliza para comparar varias 
muestras independientes (Kruskall-Wallis).

\section{Resultados}

El perfil que poseen los estudiantes en cuanto al uso de estrategias medidas por el IEEA se determina con tres niveles:

1) Puntajes menores a 11 puntos señalan la necesidad urgente de recibir entrenamiento para el desarrollo de las estrategias de autorregulación.

2) Una puntuación de 12 a 16 puntos indica que el estudiante sólo necesita apoyo para el desarrollo de la estrategia correspondiente.

3 ) Una puntuación de 17 o mayor revela que el estudiante no tiene problemas con el manejo de las estrategias de autorregulación.

Para el caso de la subescala de autorregulación persona aprobación externa $(A E)$, que es negativa, la interpretación es diferente: a) un puntaje de 5 puntos o menos, demuestra que el estudiante no tiene problemas con el manejo de las estrategias de aprobación externa; b) un puntaje de 6 a 10 puntos, prueba que el estudiante requiere apoyo para el desarrollo de las estrategias correspondientes y; c) un puntaje de 11 puntos o más, descubre la necesidad urgente de recibir entrenamiento para el desarrollo de las estrategias de la subescala.

\section{Perfil de autorregulación de los estudiantes en la unidad centro}

En la figura 5 se muestra el perfil de las estrategias de autorregulación por carrera de los estudiantes adscritos a la Unidad Regional Centro. En cuanto a la escala de autorregulación persona puede observarse que la subescalas de eficacia percibida (EP) es la que presenta una puntuación menor; los estudiantes inscritos en Tecnología Electrónica (LTE) se ubican en el rango que indica necesidad urgente de entrenamiento, el resto de las carreras de la muestra se sitúan en puntuaciones entre los 12 y 16 puntos lo que indica la necesidad de

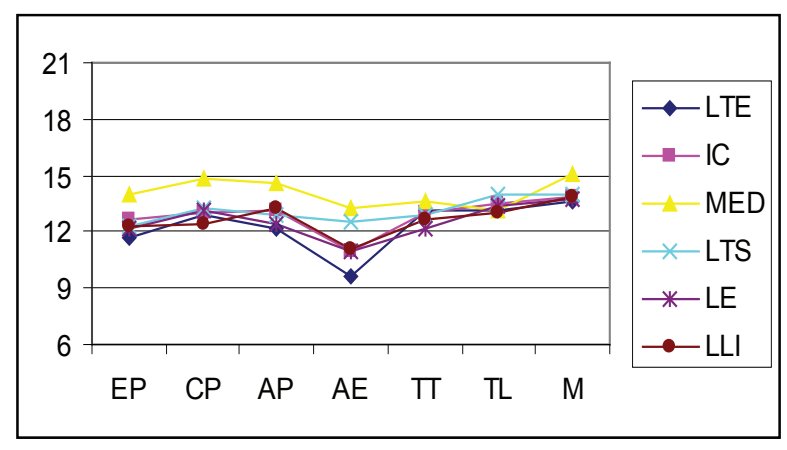

Figura 5. Perfil de estrategias de autorregulación por carrera, Unidad Regional Centro.

reforzar el resto de las subescalas, los jóvenes de Medicina (MED) presentan puntuaciones superiores al resto de los estudiantes de la muestra. En relación a la subescala aprobación externa (AE) se muestra que Medicina (MED) y Trabajo Social (LTS) se ubican por encima de los 11 puntos, lo que indica valores altos de aprobación externa, es decir, requieren entrenamiento urgente para las estrategias correspondientes.

En la escala autorregulación tarea, la subescala orientación a la tarea (TT) es la que posee una puntuación menor, aunque no se ubica en el límite inferior, los estudiantes de Economía (LE) son quienes obtuvieron una puntuación menor. En tanto que la escala de autorregulación de materiales (M) es la que posee puntuaciones más altas que las del resto de las escalas y los estudiantes de Medicina (MED) fueron quienes tuvieron puntuaciones más elevadas; el resto de las carreras de la muestra no revelan mucha variación en sus puntuaciones.

\section{Perfil de autorregulación de los estudiantes en las unidades foráneas}

La figura 6 presenta las puntuaciones que se obtuvieron por los estudiantes de las unidades foráneas en las escalas de autorregulación, en cuanto a autorregulación persona se repite el hecho de que es la subescala EP y son los estudiantes de Químico Biólogo (QBC) y 
Comunicación Organizacional (LCO) quienes presentan una puntuación por debajo de lo esperado (inferior a 12 puntos). En cuanto a la subescala $A E$ que es negativa, destaca el hecho de que los estudiantes de Ingeniería en Sistemas de Información (ISI) son los únicos que se ubican en el rango inferior, lo que demuestra un uso adecuado de las estrategias de aprobación externa.

En la escala autorregulación tarea, la subescala orientación a la tarea (TT) fueron los estudiantes inscritos en Mercadotecnia (MER) los que se colocan en una puntuación inferior a los 12 puntos, lo mismo ocurre con la subescala orientación al logro (TL), pero en este caso es acompañada de los estudiantes de Químico Biólogo (QBC). En autorregulación de materiales $(\mathrm{M})$, todas las carreras están muy cercanas unas de las otras en el rango correspondiente a la necesidad de reforzar el uso de estas estrategias, y Mercadotecnia (MER) es la que se ubica en una mejor situación.

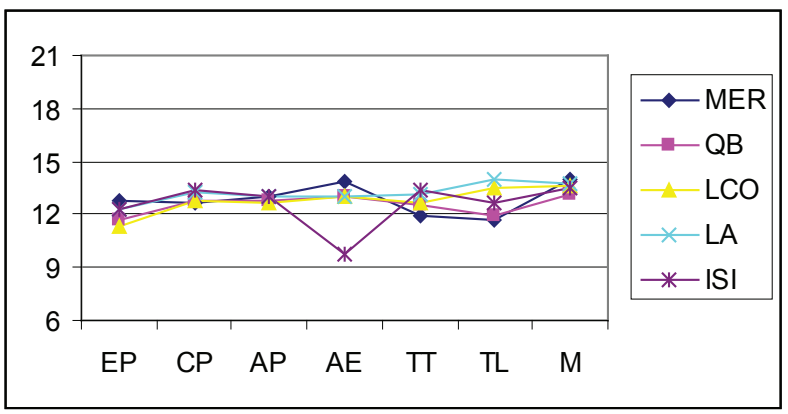

Figura 6. Perfil de estrategias de autorregulación por carrera, unidades foráneas.

\section{Comparación de la autorregulación entre las unidades}

El resultado de una comparación de medias ( $t$ test) de las escalas de autorregulación, indica que sólo dos subescalas de la escala autorregulación persona (eficacia percibida y aprobación externa) y la escala autorregulación de materiales resultaron con diferencias significativas a favor de la Unidad Centro, el resto de las escalas y subescalas de estrategias de autorregulación, también presentan medias superiores en la Unidad Centro, pero dichas diferencias no resultaron significativas.

Respecto al ranking de estrategias de autorregulación, la figura 7 refleja tales resultados donde puede señalarse que los estudiantes inscritos en Mercadotecnia (MER) se ubican a la baja en todas las escalas medidas; los estudiantes inscritos en Medicina (MED) poseen valores bajos en autorregulación de tarea con orientación al logro, escala en la que Administración (LA) posee puntuaciones mayores.

Los estudiantes de Comunicación Organizacional (LCO) presentan los valores más bajos en la escala autorregulación persona subescala eficacia percibida (APEP) y el valor mayor en esta subescala es para Mercadotecnia (MER). La Ji cuadrada resultante es de 27.23 (10 gl; $p$ < .01) para la subescala APEP; 21.71 (10 gl; $p<.05)$ para la subescala contingencia percibida

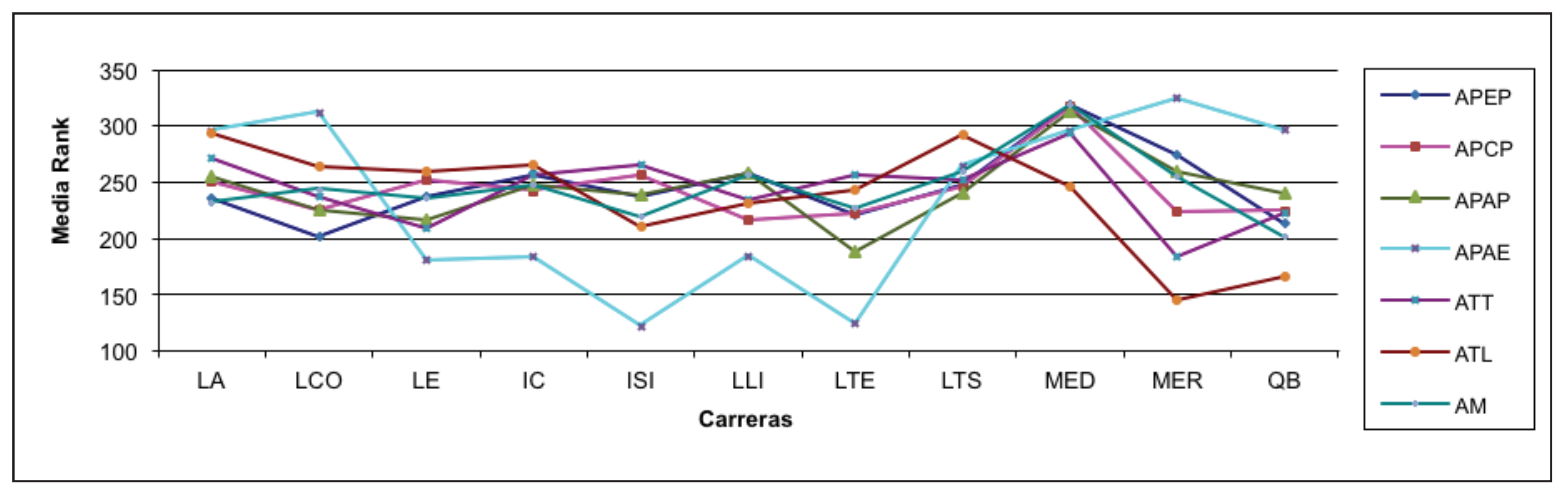

Figura 7. Puntuación media rank de estrategias de autorregulación. 
(APCP); para autonomía percibida (APAP) la Ji cuadrada fue de 22.48 (10 gl; $p<.05$ ); 98.25 (10 gl; $p$ < .01) fue el valor de Ji cuadrada para aprobación externa (APAE). Todas ellas de la escala autorregulación personal.

El valor de Ji cuadrada para autorregulación tarea con orientación a la tarea (ATT) fue de 20.88 (10 gl; $p$ < .01) y para orientación al logro (ATL) se obtuvo un valor de 45.84 (10 gl; $p<$ $.00)$, mientras que para autorregulación de materiales (AM) el valor de Ji cuadrada fue de 24.93 (10 gl; $p<.01)$.

\section{Discusión}

Puede observarse que el perfil de estrategias de autorregulación con el que ingresan los jóvenes a la universidad es similar en cuanto a que las deficiencias en subescalas son relativamente homogéneas independientemente del campus, esto se puede evidenciar al observar los resultados del contraste de medias donde se obtiene que existen diferencias significativas entre las unidades únicamente en dos subescalas de autorregulación persona como son la eficacia percibida, es decir, la eficacia que el aprendiz percibe de sí mismo para la realización de tareas académicas variadas. La otra subescala en la que se encontraron diferencias significativas fue la de aprobación externa o el deseo o interés que muestra el estudiante por obtener la aprobación de otros ante la ejecución de sus tareas académicas, sean familiares, amigos o profesores. En la escala de autorregulación de materiales también se encontraron diferencias significativas a favor de la Unidad Regional Centro.

El presente estudio no es longitudinal, sin embargo, sería más enriquecedora la comparación de medias si lo fuera, ya que existen resultados de investigación que indican que los estudiantes van desarrollando variadas y mejores estrategias de autorregulación durante su estancia en la universidad como lo encontraron por ejemplo, Martínez-Fernández
(2007) con un grupo de estudiantes de psicología con el propósito de analizar la relación de la concepción que poseen sobre el aprendizaje con el uso de estrategias metacognoscitivas; en cuanto al empleo que reportaron de estrategias cognoscitivas, encontraron que hacia el final de la carrera reportan utilizar más este tipo de estrategias en comparación con los semestres iniciales o intermedios y; González, Martínez y Corral (2004) midieron las estrategias cognoscitivas de aprendizaje y de autorregulación con un grupo de estudiantes iniciales y avanzados de seis licenciaturas y sus hallazgos reflejaron que los estudiantes de semestres avanzados refieren mayor uso de estrategias de autorregulación de persona, de tarea y de materiales en comparación con los estudiantes de semestres iniciales.

Estos resultados conducen de nuevo al concepto de aprendizaje complejo, dado que si se espera que los estudiantes sean agentes activos y responsables de sus propios aprendizajes es necesario fomentar y modelar el uso adecuado de estrategias tanto cognoscitivas como metacognoscitivas de aprendizaje, así que se apoya la opinión de Burón (1996 en Rinaudo, Chiecher \& Donolo, 2003) cuando menciona que para que una persona ponga en práctica una estrategia, es necesario que antes la conozca y sepa cuándo, cómo y por qué es pertinente usarla. En este tenor, sí se espera que el estudiante adquiera un aprendizaje significativo, además de saber qué hacer y cómo hacerlo, tendría que desarrollar estrategias de autorregulación para evaluar la forma en la que está actuando en cada situación y cómo corregir sus acciones en caso necesario.

Respecto a los resultados obtenidos sobre el perfil de estrategias de autorregulación que se refleja en los estudiantes de la muestra, se destaca que fueron los estudiantes de Medicina quienes poseen los puntajes más altos tanto en autorregulación de persona, como en autorregulación de tarea y de materiales, es 
decir, utilizan en mayor medida estrategias que les permiten evaluar, planear y regular las actividades académicas y de aprendizaje que los conducen a adquirir conocimiento tales como concentrarse en el estudio, planear sus actividades académicas y pedir ayuda cuando es necesario o bien, resolver las tareas de manera adecuada. Aspectos en los que los estudiantes de Comunicación Organizacional y Químico Biólogo requieren de entrenamiento urgente, principalmente en cuanto a eficacia percibida.

En el caso de la subescala aprobación externa los resultados muestran que todos los estudiantes requieren entrenamiento urgente, es decir, requieren fortalecer aquellas estrategias que los orienten de manera más intrínseca al aprendizaje, y no sólo resolver las tareas por aprobar sin cuidar que se logre un aprendizaje real. Estos resultados coinciden con los encontrados por Maytorena, González y Velarde (2006) quienes al estudiar a un grupo de estudiantes de licenciatura, encontraron que las estrategias de autorregulación que utilizan con mayor frecuencia son las de tarea, seguidas de las de materiales mientras que las de autorregulación persona son las menos utilizadas por los estudiantes de la muestra como ocurre también en los datos que se obtuvieron en este estudio.

En relación con los resultados de autorregulación de tarea, fue también Medicina quien se ubica en los valores más altos, sin superar el rango de necesidad de apoyo, pero en este caso, este resultado es compartido (aunque en menor medida) por los estudiantes de Economía, Químico Biólogo Comunicación Organizacional y quienes menos puntos obtuvieron en esta dimensión, específicamente enorientación ala tarea, fueron los estudiantes de Mercadotecnia del campus Caborca. Respecto a las estrategias orientadas a la autorregulación de sus materiales de aprendizaje, todos los estudiantes requieren entrenamiento (pero no urgente) en la realización de actividades tales como la organización y clasificación de los materiales de sus asignaturas; sin embargo, se considera que es debido a la novedad de sus estudios universitarios, ya que estaban en primer semestre en el momento de la evaluación, además de que puede ser también una actividad que es mediada por los profesores, pues al demandar a los estudiantes ejercicios y actividades propias de la asignatura que imparten es posible también moldear y modelar la organización de los materiales de aprendizaje utilizados por los estudiantes.

Los resultados encontrados en este trabajo de investigación permiten señalar la importancia de una enseñanza integral en la que el interés fundamental no sea solamente la adquisición de un cúmulo de conceptos, principios, reglas y procedimientos, sino también fomentar en los estudiantes el desarrollo de habilidades que además de permitirles seguir aprendiendo, les posibilite regular su aprendizaje y el uso que van a hacer de él con el fin de establecer metas claras y orientadas hacia su desarrollo profesional y personal. Aprender de manera adaptada, es encontrar un balance entre dos prioridades paralelas que permiten extender los recursos personales disponibles y prevenir la pérdida de los recursos y la distorsión de su bienestar (Cárdenas, 2006).

\section{Conclusiones}

Los resultados que se encontraron en estos estudios, conducen a la necesidad existente en la educación superior de atender las características que los estudiantes poseen al ingresar a una institución de educación superior. Los exámenes de admisión no son determinantes en este aspecto puesto que además de conocimientos se requieren otras habilidades importantes para el aprendizaje y poseer características que ayuden a la adquisición de nuevas habilidades y conocimientos como es el caso de las variables que se midieron en este estudio.

En relación con las estrategias cognoscitivas 
de aprendizaje y las estrategias de autorregulación que se han investigado desde hace aproximadamente dos décadas, particularmente en la Universidad Nacional Autónoma de México, se han desarrollado instrumentos orientados a la evaluación y el fomento de tales estrategias, por este emotivo son el instrumento utilizado en este estudio. Desde el punto de vista de la investigación es una gran ventaja contar con este tipo de instrumentos desarrollados en nuestro país, ya que ello lleva a la posibilidad de poseer instrumentos con validez ecológica que faciliten identificar las características de nuestros estudiantes mexicanos.

Mientras que desde la perspectiva de la psicología aplicada, este tipo de trabajos y desarrollos tecnológicos brindan la posibilidad de un entrenamiento específico tanto a estudiantes como a profesores, en opinión de Castañeda, Pineda, Gutiérrez y Romero (2010) "atender los procesos asociados al aprendizaje podrían conducir a la identificación y solución de problemas relacionados con la autonomía y la efectividad de los estudiantes en sus procesos de construcción de conocimiento, aspectos centrales en entornos de alta competitividad en los que se requieren profesionales expertos" (p. 83).

Estos resultados y la necesidad aún vigente de atender los procesos de aprendizaje tanto en investigación como en la aplicación de las ciencias educativas hacen pertinente referir a Straka quien en 1997 señaló que el aprendizaje en la vida diaria, en la empresa y en la escuela se realiza en un continuo entre hetero y autodirección, en el que, en la aplicación práctica, ambos polos no aparecen.

Estos resultados señalan la necesidad expresada anteriormente acerca de que convertirse en un estudiante autorregulado enfatiza lo errado de asumir un tipo de innatismo para el origen de las destrezas necesarias para transformarse en un aprendiz autorregulado por lo que queda clara la necesidad de un rol diferencial de la enseñanza, es decir, si se asume la importancia del rol activo de los estudiantes en el proceso de aprendizaje, esto conduce directamente a la conclusión de que los profesores son agentes importantes en este proceso y deben ser partícipes de la enseñanza de estas estrategias (Revel \& González, 2007).

Lo expresado por Revel y González (op. cit.) va en concordancia con lo dicho por Wittrock años atrás (1998) acerca de que la efectividad instruccional se puede mejorar a través de instar a los profesores a que incorporen variables cognoscitivas y metacognoscitivas al ejercicio de la planeación, conducción y evaluación informal en su trabajo cotidiano, y que estén atentos y reflexionen acerca de cómo sus secuencias instruccionales les permitirán alcanzar sus objetivos. También se recomienda asumir que la enseñanza no afecta directamente al logro académico, ni es un efecto automático; sino más bien, que esta influencia se da en forma indirecta, mediada por la estructura de conocimientos del estudiante, el uso que haga de sus estrategias de aprendizaje y de sus procesos metacognoscitivos y metamotivacionales.

Estas medidas de motivación han indicado la necesidad de atender los aspectos motivacionales de los estudiantes, principalmente al ingresar a la universidad tanto en los contenidos curriculares como en las estrategias de evaluación (Aguilar, Valencia, Martínez \& Vallejo, 2002). Por lo que se refleja la necesidad de atender con más ahínco los procesos motivacionales con que cuentan los estudiantes al ingresar a la universidad e irlos orientando hacia los propósitos y metas 


\section{asociadas al estudio de una carrera universitaria.}

\section{Referencias}

Abril, E., Román, R. y Cubillas, M. (2002). Educación y empleo en jóvenes sonorenses. Investigaciones Educativas en Sonora, 4, 229-243.

Aguilar, J., Pacheco, J., Andrade, J., Vargas, A., Gutiérrez, P. y Zetina, G. (1992). Estudio sobre la validez concurrente del Inventario de Factores de Carrera en estudiantes de licenciatura. Monografía. México: UNAM.

Aguilar, J., Peña, L., Pacheco, J. y De la Paz, C. (1993). Adaptación y ampliación del Inventario de factores de carrera. Investigación Psicológica, 3, 53-63.

Aguilar, J. \& Valencia, A. (1994). Medición e interrelaciones entre temor al fracaso y morosidad. Revista de Psicología Social y Personalidad, 10, 145-155.

Aguilar, J., Valencia, A., Martínez, M. y Vallejo, A. (2002). Un modelo estructural de la motivación extrínseca en estudiantes universitarios. En A. Bazán y A. Arce (eds.). Estrategias de Evaluación y Medición del Comportamiento en Psicología (pp. 165-185). Cd. Obregón, Sonora: ITSON - UADY

Araujo, V., Cerezo, S., Coronado, O. y Hernández, M. (2008) Desempeño académico de universitarios en relación con ansiedad escolar y autoevaluación. Acta Colombiana de Psicología, 11, 13-23.

Backhoff, E. y Tirado, F. (1992). Desarrollo del examen de habilidades y conocimientos básicos (EXHCOBA). Revista de la Educación Superior, 83, 95-117.

Backhoff, E. y Tirado, F. (1993). Habilidades y conocimientos básicos del estudiante universitario: hacia los estándares nacionales. Revista de la Educación Superior, 88, 45-65.

Backhoff, E. y Tirado, F. (1994). Estructura y lógica del examen de habilidades y conocimientos básicos (EXHCOBA). Revista Sonorense de Psicología, 8, 21-33.

Canto y Rodríguez, J.E. (2000). Certeza vocacional y autoeficacia en la elección de carrera y ambiente familiar. Enseñanza e Investigación en Psicología, 5, 1, 51-71.

Carbonero, M. y Navarro, J. (2006). Entrenamiento de alumnos de educación superior en estrategias de aprendizaje en matemáticas. Psicothema, 18, 348-352.

Cárdenas, B. (2006). La Influencia de la Motivación y la Autorregulación en el Desempeño Académico Universitario. Tesis de Licenciatura en Psicología. Universidad de Sonora.

Castañeda, S. (1998). Evaluación y fomento del desarrollo intelectual en la enseñanza de ciencias, artes y técnicas: Perspectiva internacional en el umbral del siglo XXI. México: Porrúa-UNAM.

Castañeda, F. S., Lugo, E., Pineda, L. y Romero, N. (1998). Estado del Arte de la evaluación y el fomento del desarrollo intelectual en la enseñanza de ciencia, artes y técnicas. En S. Castañeda (Coord.), Evaluación y Fomento del Desarrollo Intelectual en la Enseñanza de Ciencias, Artes y Técnicas: Perspectiva internacional en el umbral del siglo XXI. (17-158). México: Porrua-CONACYT-UNAM.

Castañeda, S. (2002). Educación, aprendizaje y cognición. En S. Castañeda (ed.). Educación, Aprendizaje y Cognición. Teoría en la práctica (pp. 49-74). México: El Manual Moderno.
Castañeda, S. (2003). Inventario de Estrategias de Estudio y Autorregulación (IEEA). Manuscrito no publicado. México: UNAM.

Castañeda, S., Pineda, M., Gutiérrez, E. y Romero, N. (2010) Construcción de instrumentos de estrategias de estudio, autorregulación y epistemología personal. Validación de constructo. Revista Mexicana de Psicología, 27, 77-85.

Castañeda, S. y Martínez, R. (1999). Enseñanza y aprendizaje estratégicos. Modelo integral de evaluación e instrucción. Revista Latina de Pensamiento y Lenguaje, 4, 251-278.

Castañeda, S. y Ortega, I. (2004). Evaluación de estrategias de aprendizaje y orientación motivacional al estudio. En S. Castañeda (ed.) Aprendizaje y Cognición. Teoría en la práctica (pp. 277-299). México: Manual Moderno.

Corral, V., Frías, M., Pavlovich, S., Ibarra, D., Mejía, K., Rolón, L., Valdez, G. y Velarde, D. (2003). Correlatos de la conducta antisocial y del aprovechamiento escolar en estudiantes de secundaria: propensión al futuro, uso de la Internet y variables demográficas. Investigaciones Educativas en Sonora, 5, 55-70.

Cupani, M. y Pérez, E. (2006). Metas de elección de carrera: contribución de los intereses vocacionales, la autoeficacia y los rasgos de personalidad. Interdisciplinaria, 23, 81-100.

De Corte, E. (1999). Desarrollo cognitivo de innovación tecnológica. Una nueva concepción de la enseñanza y el aprendizaje para el siglo XXI. Revista Latina de Pensamiento y Lenguaje, 4, 229-250.

Díaz-Morales, J. (2006). Estructura factorial y fiabilidad del Inventario de Perspectiva Temporal de Zimbardo. Psicothema, 18, 565-571.

González-Lizárraga, G. (1999). Perfil de ingreso de los alumnos de la Universidad de Sonora, 1997-1998. En J. Ramos (comp.). Investigación Educativa en Sonora (pp. 132-139). México: Editorial UNISON.

González, D. (2002). Desempeño Académico Universitario: variables psicológicas. Sonora, México: Editorial Uni-Son.

González, D., Castañeda, S. y Maytorena, M. (2000). Estilos de aprendizaje y aprovechamiento en ingresantes universitarios. Revista de Psicología, 18, 199-225.

González, D., Castañeda, S. y Corral, V. (2002). Validación e Identificación de Constructos Subyacentes a Estrategias de Aprendizaje Universitario: Aproximación Multirasgo-Multimétodo (MRMM). Revista Latina de Pensamiento y Lenguaje, 10, 107-118.

González, D., Martínez, M. \& Corral, V. (2004). Estrategias de aprendizaje y autorregulación en estudiantes novatos y avanzados. En E. Carlos, J. Ramos y L. Galván (comps.). Anuario de Investigaciones Educativas, volumen 6 (pp. 137-143). Hermosillo, Sonora: REDIESCIAD-SEC.

González, D. y Maytorena, M. (en prensa). Certeza vocacional en estudiantes universitarios y de bachillerato: ocho años de evidencias. Revista Decisión Empresarial para su edición especial "Educación".

González, D. y Maytorena, M. (2003). Decisión vocacional en estudiantes de bachillerato y educación superior. Revista Sonorense de Psicología, 17,43-50.

González, D. y Maytorena, M. (2005). Modelo estructural de factores de carrera, seguridad vocacional y esfuerzo académico. Revista Interamericana de Psicología, 39, 1-9. Brasil. 
González, D., Maytorena, M., Lohr, F.y Carreño, E. (2006). Perspectiva temporal y morosidad académica en estudiantes universitarios, Revista Colombiana de Psicología, 15, 15-24.

López-Olivas, M. (2007). El modelo de alumno deseable para la institución ¿es el que refleja el modelo de examen de admisión? En D. González y M. Maytorena (eds.). Estudios empíricos en educación superior (pp. 19-35). México: UNISON.

Martínez-Fernández, R. (2007). Concepción de aprendizaje y estrategias metacognitivas en estudiantes universitarios de psicología. Anales de Psicología, 23, 7-16.

Maytorena, M., González, D. y Velarde, D. (2006). Estrategias de aprendizaje y certeza vocacional en estudiantes de tres licenciaturas. Congreso Mexicano de Psicología Social, XI (pp. 528-534). México: AMEPSO.

Maytorena, M. y González, D. (2008). Estrategias de aprendizaje y de autorregulación en estudiantes de licenciatura: seguimiento. La Psicología Social en México, XII, 707-712. México: AMEPSO.

Pintrich, P. (1998). El papel de la motivación en el aprendizaje académico autorregulado. En S. Castañeda (ed.). Evaluación y Fomento del Desarrollo Intelectual en la Enseñanza de Ciencias, Artes y Técnicas. Perspectiva internacional en el umbral del Siglo XXI (pp. 229-262). México: UNAM-CONACYT-PORRÚA.

Pintrich, P. \& García, T. (1991). Student goal orientation and selfregulation in the college classroom. En M. Maehr y P. Pintrich (eds.). Advances in Motivation and Achivement: Goals and self-regulatory processes ( pp. 371-402). Greenwich, CT: JAI.

Piña-Osuna, F. (2011). Conocimientos básicos de bachillerato a partir del EXHCOBA y mejoramiento del desempeño académico en universitarios. IV Reporte de Avance de Investigación de la Maestría en Innovación Educativa, de la Universidad de Sonora.

Revel, C. y González, L. (2007). Estrategias de aprendizaje y autorregulación. Revista Latinoamericana de Estudios Educativos, 3, 2, pp. 87-98. Recuperado de www.redalyc.org el 30 de junio del 2010.

Rinaudo, M., Chiecher, A. y Donolo, D. (2003). Motivación y uso de estrategias en estudiantes universitarios. Su evaluación a partir del Strategies Learning Questionarie. Anales de Psicología, 19, 107-119.

Sociedad Mexicana de Psicología. (2010). Código Ético del Psicólogo. México: CNEIP-SMP-Trillas.

Suárez, J. y Fernández, A. (2005). Escalas de evaluación de las estrategias motivacionales de los estudiantes. Anales de psicología, 21, 001, 116-128. Recuperado el 15 de junio de: www.redalyc.org.

Straka, A., Nenninger, P., Spevacecek, G. y Wosnitza, M. (1997). Un modelo de aprendizaje motivado y autorregulación. Educación: aportaciones alemanas, 55, 43- 54.

Velarde, D., González, D. y Maytorena, M. (2006). Estilos de aprendizaje, orientación motivacional y certeza vocacional. En R. Sánchez, R. Díaz-Loving (eds.). La Psicología Social en México, volumen XI (528-534). México: AMEPSO-Universidad Juárez Autónoma de Tabasco.

Wittrock, C. (1998). Aplicación de pruebas e investigación reciente en cognición. En M. Wittrock y E. Baker (comps.). Test y Cognición: Investigación cognitive y mejora de las pruebas psicológicas (pp. 1720). Barcelona: Paidós Ibérica.

Zimbardo, P. \& Boyd, J. (1999). Putting Time in Perspective: a valid, reliable individual diferences metric. Journal of Personality and Social Psychology, 66, 742-752.

\section{PSICUMEX}

